\title{
The effect of adapting Hospital at Home to facilitate implementation and sustainment on program drift or voltage drop
}

\author{
Albert L. Siu ${ }^{1,5^{*}}$ (D), Robert M. Zimbroff ${ }^{2}$, Alex D. Federman ${ }^{3}$, Linda V. DeCherrie ${ }^{1}$, Melissa Garrido ${ }^{6}$, Barbara Morano ${ }^{1}$,
} Sara Lubetsky ${ }^{1}$, Elisse Catalan ${ }^{1}$ and Bruce Leff ${ }^{4}$

\begin{abstract}
Background: Translating evidence-based interventions from study conditions to actual practice necessarily requires adaptation. We implemented an evidence-based Hospital at Home $(\mathrm{HaH})$ intervention and evaluated whether adaptations could avoid diminished benefit from "voltage drop" (decreased benefit when interventions are applied under more heterogeneous conditions than existing in studies) or "program drift." (decreased benefit arising from deviations from study protocols).

Methods: Patients were enrolled in $\mathrm{HaH}$ over a 6-month pilot period followed by nine quarters of implementation activity. The program retained core components of the original evidence-based HaH model, but adaptations were made at inception and throughout the implementation. These adaptations were coded as to who made them, what was modified, for whom the adaptations were made, and the nature of the adaptations. We collected information on length of stay (LOS), 30-day readmissions and emergency department (ED) visits, escalations to the hospital, and patient ratings of care. Outcomes were assessed by quarter of admission. Selected outcomes were tracked and fed back to the program leadership. We used logistic or linear regression with an independent variable included for the numerical quarter of enrollment after the initial 6-month pilot phase. Models controlled for season and for patient characteristics.
\end{abstract}

Results: Adaptations were made throughout the implementation period. The nature of adaptations was most commonly to add or to substitute new program elements. HaH services substituting for a hospital stay were received by 295 patients (a mean of 33, range 11-44, per quarter). A small effect of quarter from program inception was seen for escalations (OR 1.09, 95\% Cl 1.01 to $1.18, p=0.03$ ), but no effect was observed for LOS ( -0.007 days/quarter; SE 0.02, $p=0.75), 30$ day ED visit (OR 0.93, 95\% Cl 0.86 to $1.01, p=0.09$ ), 30-day readmission (OR $1.00,95 \% \mathrm{Cl} 0.93$ to $1.08, p=0.99$ ), or patient rating of overall hospital care (OR for highest overall rating $0.99,95 \% \mathrm{Cl} 0.93$ to $1.05, p=0.66$ ).

Conclusions: We made adaptations to $\mathrm{HaH}$ at inception and over the course of implementation. Our findings indicate that adaptations to evidence-based programs may avoid diminished benefits due to potential 'program drift' or 'voltage drop.'

Trial registration: Not applicable. This study is not a clinical trial by the International Committee of Medical Journal Editors (ICMJE) definition because it is an observational study "in which the assignment of the medical intervention is not at the discretion of the investigator."

Keywords: Implementation, Adaptation, Health outcome assessment, Quality of care

\footnotetext{
* Correspondence: albert.siu@mountsinai.org

${ }^{1}$ Brookdale Department of Geriatrics and Palliative Medicine, Icahn School of Medicine at Mount Sinai, 1 Gustave L. Levy Place, New York, NY 10029, USA

5 James J. Peters VA Medical Center, New York, NY, USA

Full list of author information is available at the end of the article
}

(c) The Author(s). 2019 Open Access This article is distributed under the terms of the Creative Commons Attribution 4.0 International License (http://creativecommons.org/licenses/by/4.0/), which permits unrestricted use, distribution, and reproduction in any medium, provided you give appropriate credit to the original author(s) and the source, provide a link to the Creative Commons license, and indicate if changes were made. The Creative Commons Public Domain Dedication waiver (http://creativecommons.org/publicdomain/zero/1.0/) applies to the data made available in this article, unless otherwise stated. 


\section{Background}

Translating complex multi-component evidence-based interventions from study conditions and protocols to actual practice necessarily requires minor to major adaptation of intervention content, format, personnel roles and processes. Adaptations may be desired or necessary to customize to local circumstances or to account for new technology or temporal changes in disease epidemiology or clinical practice [1, 2]. Traditionally, it has been thought that variance from an evidence-based protocol is accompanied with at least some diminution of effectiveness. This effect has been termed "program drift" (i.e., decreased benefit arising from deviations from study protocols) and "voltage drop" (i.e., loss of benefit when interventions are applied in more heterogeneous patient populations and settings as they move from efficacy to effectiveness and into actual practice) [3].

More recently, the inevitability of diminished benefits due to program drift and voltage drop has been questioned. While fidelity to a study population, setting, and protocol may be critical for a biological or a clinical intervention to maintain the studied benefits, this may not be the case for health system interventions or more complex multi-component interventions. Adaptations may be made as the intervention is applied in more heterogenous patient populations or practice settings, thereby potentiating voltage drop. Other adaptations may be made to the intervention protocol to improve fit with the practice setting and the environment, thereby potentiating program drift. Other adaptations may address technological or medical advances that were not available or formally manualized at the time of the evidence-based study. In theory, these adaptations may have neutral effects. Some adaptations may enhance the effectiveness of the original intervention $^{3,}$ thereby neutralizing any diminished effectiveness from program drift or voltage drop or perhaps even increasing the net benefit beyond that observed in evidence-based studies. Other adaptations may inadvertently diminish benefits. Indeed, some have called for the systematic study of adaptations to evidence-based interventions and a formalized structure for updating the evidence base to account for adaptation [4].

To improve our understanding of the effect of adaptations, we took advantage of an implementation of an evidence-based hospital admission avoidance Hospital at Home $(\mathrm{HaH})$ intervention [5]. This implementation necessitated extensive adaptation of studied procedures to local setting and culture and evolving medical practice. In 2014, The Mount Sinai Health System, a seven-hospital system in New York City, began implementing $\mathrm{HaH}$ with 30-day post-acute care follow-up of patients. For select patients with specific diagnoses (e.g., pneumonia) who would otherwise be admitted, $\mathrm{HaH}$ services (e.g., intravenous antibiotics, fluids, oxygen, etc.) and daily clinician visits are provided at home along with durable medical equipment, phlebotomy, and home x-ray as needed. We examined whether $\mathrm{HaH}$ associations with outcomes changed over time as adaptations were put into place.

Funded as an innovation award from the Center for Medicare and Medicaid Innovation, this implementation of $\mathrm{HaH}$ was instigated with the goal of eventual sustainment in the local health system and scaling and dissemination nationally. The phased plan for implementation within the local health system was consistent with the concepts outlined in the Dynamic Sustainability Framework (DSF) [3]. The DSF posits that interventions should be implemented with consideration of fit with practice settings and the external ecological system of competitors, regulation, market forces, and population needs. The framework also puts forth that these elements are not static and will change with time. Our phased implementation anticipated a piloting phase followed by initial implementation in one hospital emergency department with phased expansion over several quarters to other health system settings. We anticipated the need to adapt $\mathrm{HaH}$ to each hospital's unique culture, medical staff, electronic record system, community, and labor practices. Similarly, we anticipated that $\mathrm{HaH}$ might need to be adapted to changing policy and regulations, different payers, and a healthcare market in dynamic flux due, in part, to the implementation of the Affordable Care Act.

\section{Methods}

\section{Patients and settings}

Patients were enrolled in $\mathrm{HaH}$ starting in November 2014 for 33 months through August 2017. An initial 6-month run-in pilot period was followed by nine quarters of implementation activity.

Details of $\mathrm{HaH}$ patient engagement procedures have been reported elsewhere [6]. Briefly, potentially eligible patients were identified in the emergency departments of Mount Sinai Health System hospitals, or by referral from physicians in outpatient clinical practices or a home-based primary care practice.

Patients were eligible for $\mathrm{HaH}$ if they were $\geq 18$ years of age, required inpatient admission, and had fee-for-service Medicare or coverage from a single private insurer that contracted with Mount Sinai for $\mathrm{HaH}$ services. Patients were excluded if they were clinically unstable (e.g., had very low blood pressure), required cardiac monitoring or intensive care, lived in an unsafe home environment, or resided outside the specified catchment area.

\section{Hospital at Home, Core components, and adaptations and their coding}

The $\mathrm{HaH}$ program retained many of the core components of original evidence-based studies of $\mathrm{HaH}$ 


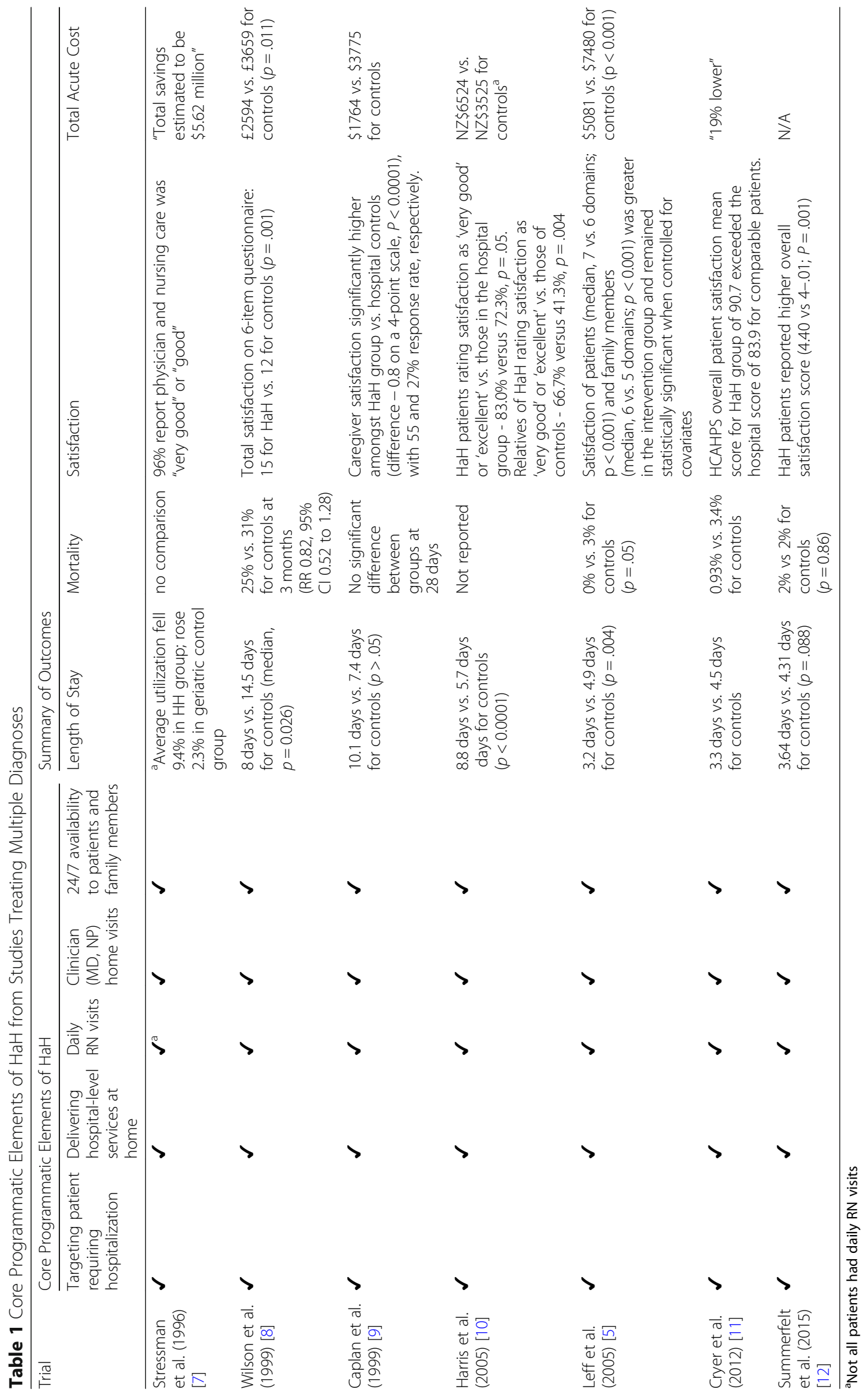


(see Table 1). These included a) targeting to participants who needed to be hospitalized; b) delivering hospital-level services at home instead of the hospital; c) daily registered nurse visits to the home; d) clinician (physician or nurse practitioner) home visits; and e) 24/7availability to patients and family members.

Adaptations to the original $\mathrm{HaH}$ model were made at inception and throughout the 33-month implementation to enhance enrollment, to add new hospital sites, to improve workflow, to enhance patient care, to extend hours of intake, and to take advantage of new opportunities (availability of new technology or services such as community paramedicine). As described later in the list of adaptations made to the $\mathrm{HaH}$ program, $\mathrm{HaH}$ service lines evolved to include palliative care at home, observation unit at home, and rehabilitation at home (see full list in Table 2). We used an established coding system to categorize who made the adaptations, what was modified, for whom the adaptations were made, and the nature of the adaptations [1].

\section{Data collection and measures}

Patient-level data were collected for the purposes of performance monitoring and final evaluation of program performance. Interviews were conducted at the bedside in

Table 2 Adaptations of $\mathrm{HaH}$ Model

\begin{tabular}{ll}
\hline Adaptations & Rationale \\
\hline $\begin{array}{l}\text { Addition of 30-day post-acute transition component } \\
\text { to the HaH model }\end{array}$ & $\begin{array}{l}\text { To improve transitions of care, reduce preventable } \\
\text { readmissions, and establish follow up with primary } \\
\text { care }\end{array}$ \\
$\begin{array}{l}\text { Expansion of original target diagnoses and reduce } \\
\text { exclusions (e.g., HIV exclusion) to reflect current }\end{array}$ & $\begin{array}{l}\text { To enroll patients with a broader set of diagnoses } \\
\text { medical practice }\end{array}$ \\
$\begin{array}{l}\text { judgment } \\
\text { Implementation of Palliative Care Unit at Home }\end{array}$ & $\begin{array}{l}\text { To provide acute services at home consistent with } \\
\text { stated goals of care for patients with advanced illness } \\
\text { who would otherwise have been excluded from HaH }\end{array}$
\end{tabular}

Collaborated with community paramedicine program to consult with $\mathrm{HaH}$ physicians by video for patients needing urgent visits in the home

Contracting for infusion services

Dedicated nurses hired

Implementation of Observation at Home

Implementation of Rehabilitation at Home

Expansion to new sites for enrollment along with developing new intake procedures customized for each site

Adaptation of intake procedure for patients identified to need $\mathrm{HaH}$ services late at night by holding the patients overnight in the emergency department and transferring home in the morning

Launch of telehealth visits to supplement home visits

Internalized major portions of pharmacy and lab services

Implemented new version of electronic medical record

Dedicated physical therapist hired

Role created for nurse care coordinator

Piloted weekend admissions
To better evaluate and address urgent clinical needs and avoid unnecessary visits to the emergency department

1 then suspended due to bankruptcy of partner and restarted in 6 with new partner

To increase staffing flexibility in being to provide infusion services

To increase availability and consistency of nursing staff for the program

To treat patients with observation services at home with the expectation that some of these patients would require more extended $\mathrm{HaH}$ services

To treat patients who would otherwise require admission to a subacute rehabilitation facility in the home setting

2

3

To capture and enroll patients presenting to the ED overnight

To increase the frequency and efficiency of clinician contacts in the home

To speed availability of services to be provided to patients in the home

To update an earlier version of a $\mathrm{HaH}$-specific electronic medical record to improve documentation and communication

To increase availability and consistency of physical therapy services for the program

To triage patient needs and coordinate staff involved 8 in home visits

To increase service hours 8 
the emergency department (ED) (see Additional file 1 for instrument guide), with follow-up interviews conducted at 2 and 4 weeks after admission by phone (see Additional files 2 and 3 for instrument guide). We collected information on the following patient outcomes: length of stay (LOS), 30-day readmissions and emergency department (ED) visits, escalations (i.e., needing to suspend a $\mathrm{HaH}$ episode in the acute rather than post-acute phase to transfer the patient to the hospital), and patient ratings of care measured with the Hospital Consumer Assessment of Healthcare Providers and Systems (HCAHPS) survey. HCAHPS was scored per guidelines from the Centers for Medicare and Medicaid Services [13], specifically, the proportion of individuals who gave a top-box rating for the measure (highest possible rating), adjusted for age, education, interview language (Spanish or English), and general health. Interviewers also documented patient demographics, performance in 5 activities of daily living (ADL), and general health (rated poor to excellent). Functional impairment was defined as needing some help or unable to perform one or more of 12 ADLs. Data on all outcomes, with the exception of patient ratings, which were less available to us in a timely fashion, were monitored monthly with feedback to program staff for review and continuous quality improvement.

\section{Analysis}

Because the outcomes collected were most relevant to hospital stays, we report outcomes for patients admitted to the program where the intervention was used to substitute for a hospital stay. That is, we excluded patients receiving rehabilitation at home $(n=264)$. We also exclude patients who received observation services for a single overnight period $(n=41)$. If hospital services were extended overnight past a second midnight for patients initially admitted to observation services, we considered the case to have been converted into a regular $\mathrm{HaH}$ stay and include them in our analyses; this simulates what would have happened if a patient had been admitted to a regular hospital observation unit and required services past the second midnight. Patients receiving palliative care services at home were receiving $\mathrm{HaH}$ for what substitutes as a hospital stay and are included as well. After exclusions, our sample includes data on 295 patients.

We present outcomes by quarter of $\mathrm{HaH}$ patient admission. We used logistic or linear regression (in the case of LOS) with an independent variable included for the numerical quarter of enrollment after an initial 6-month pilot phase. We estimated the average marginal effect for number of quarters since the program began (marginal effect per each additional quarter) for LOS. Odds ratios were estimated for the other models. The models controlled for season of $\mathrm{HaH}$ admission, patient age, sex, race, ethnicity, education, insurance type, functional impairment, and self-rated health.

More than $10 \%$ of data were missing for race and ethnicity $(n=64)$, physical function $(n=115)$, and general health $(n=84)$. To maximize the number of patients in the models, we used multiple imputation, modeling the probability of missing data for each variable on age, sex, race and ethnicity, education, insurance type, pre-acute physical function, and general health. Analyses were performed with SAS version 9.3 (SAS Institute, Cary, NC).

Because the data in these analyses were used for internal program evaluation and reporting to Medicare, their collection was exempt from Mount Sinai Institutional Review Board (IRB) review and patient consent was not required. We requested and received approval from our IRB to conduct a retrospective analysis of these data.

\section{Results}

Over the 33 months of implementation, a series of program adaptations were implemented (see Table 2), including a) adding a 30-day post-acute transition component to the $\mathrm{HaH}$ model (at inception); b) expanding target diagnoses and modifying exclusions from those originally studied (Quarter 1) due to changes in medical practice (e.g., removed HIV exclusion from decade-old protocol of original $\mathrm{HaH}$ studies); c) outside contracting for certain services (e.g., infusion) rather than providing the service directly with program staff (Quarter 2); d) implementing $\mathrm{HaH}$ variations due to change in medical practice (e.g., palliative care unit at home [Quarter 1], observation unit services at home [Quarter 3]) or opportunity (e.g., rehabilitation at home services [Quarter 3]); e) adapting intake procedures (e.g., holding patients overnight in the emergency room [Quarter 4]) for patients entering the program late at night when delivering supplies to the home to initiate a $\mathrm{HaH}$ episode was less available; $f$ ) launching telehealth visits (Quarter 4); and g) changing staffing and staff roles (Quarters 2, 6, 8). Other more minor adaptations were made throughout the implementation period.

All adaptations were initiated by the program team (see Table 3). Modifications were most commonly made in format (or how the intervention was delivered) and in personnel and roles. Modifications were made at the level of the cohort (e.g., for patients who would otherwise have been admitted to an observation unit in the hospital), patient population (e.g., for patients with new diagnoses or with palliative care needs that would not have been eligible for the original $\mathrm{HaH}$ intervention) or organization (e.g., staffing modifications that affected the entire program regardless of hospital site). The nature of adaptations was most commonly to add or to substitute new program elements. In three instances, these adaptations were sufficiently extensive for the cohort or 


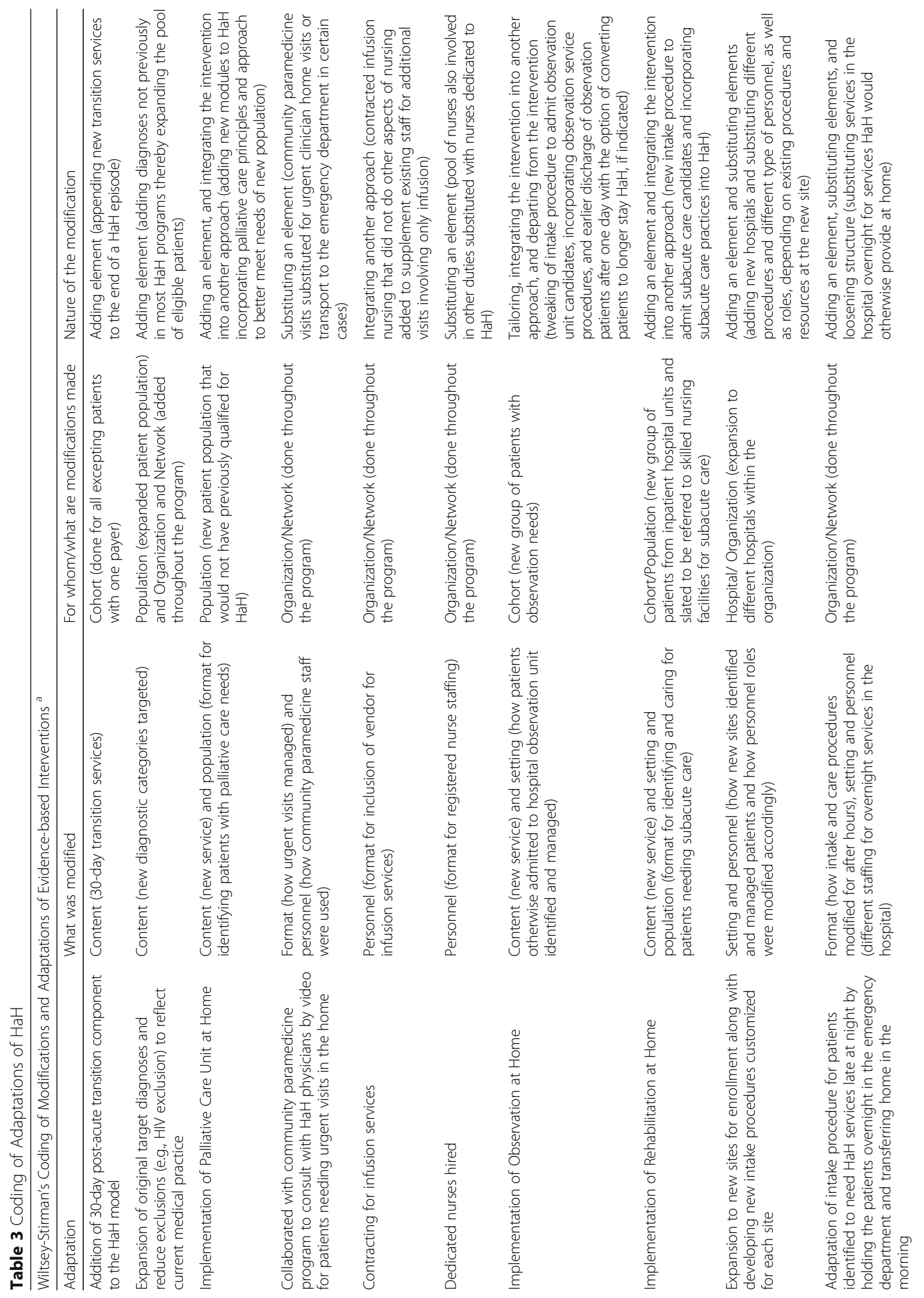




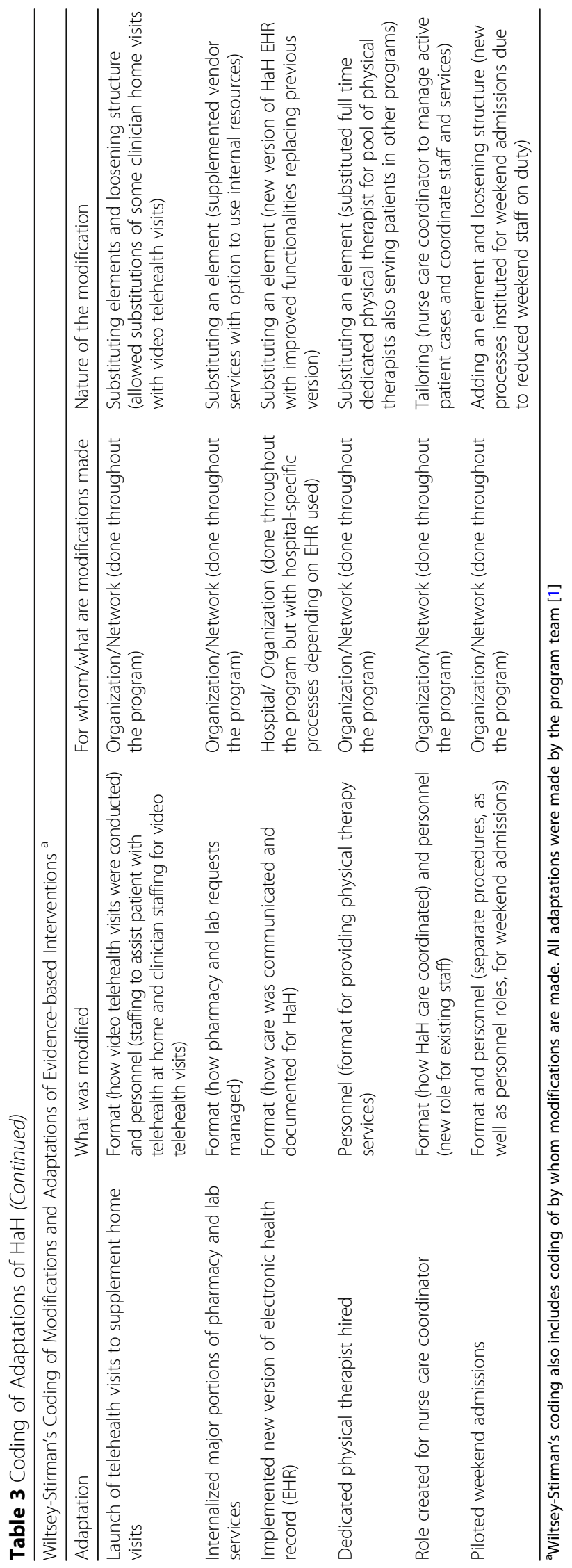


population that it involved integrating the $\mathrm{HaH}$ approach to additional patient populations (palliative care patients) or to other clinical constructs (e.g., observation unit services or postacute rehabilitation).

$\mathrm{HaH}$ services substituting for a hospital stay were received by 295 patients. The average LOS was 3.2 days. Of our sample, $12.2 \%$ had escalations, $8.6 \%$ had readmissions within 30 days, and $5.8 \%$ had ED visits within 30 days. The overall rating for hospital care was rated at the highest level by $68.8 \%$ of patients.

Graphs of these outcomes, adjusted for patient characteristics and season, over the nine quarters of implementation are provided in Figs. 1, 2, 3 and 4. A median of 33 patients (range 11-44) received services each quarter. The graphs show occasional spikes in events such as readmissions. Confidence intervals and standard errors were moderately large at any point in time; however they indicate that changes, if any, were small over time. The graphs indicate the quarter of initiation for the adaptations listed in Tables 2 and 3.
Regression models controlling for patient characteristics and season of admission are available in Additional file 4. Older age was associated with higher ED revisits and higher ratings of care. African American patients had shorter LOS and higher 30-day ED revisits and readmissions. Having Medicaid was associated with higher ED revisits and lower ratings of care. Poor self-rated health was associated with longer LOS, greater escalations, and higher ratings of care.

In the regressions, we did not observe a relationship between quarter from program inception and LOS $(-0.007$ days/quarter; SE 0.02, $p=0.75)$. The odds ratios (OR) per quarter from program inception was mildly increased for escalations (OR 1.09, 95\% CI 1.01 to $1.18, p=0.03$ ), but was not increased or decreased for 30 day ED visit (OR $0.93,95 \%$ CI 0.86 to 1.01 , $p=0.09$ ), 30-day readmission (OR 1.00, 95\% CI 0.93 to $1.08, p=0.99)$, and the patient providing the highest rating for overall hospital care (OR 0.99, 95\% CI 0.93 to $1.05, p=0.66)$.

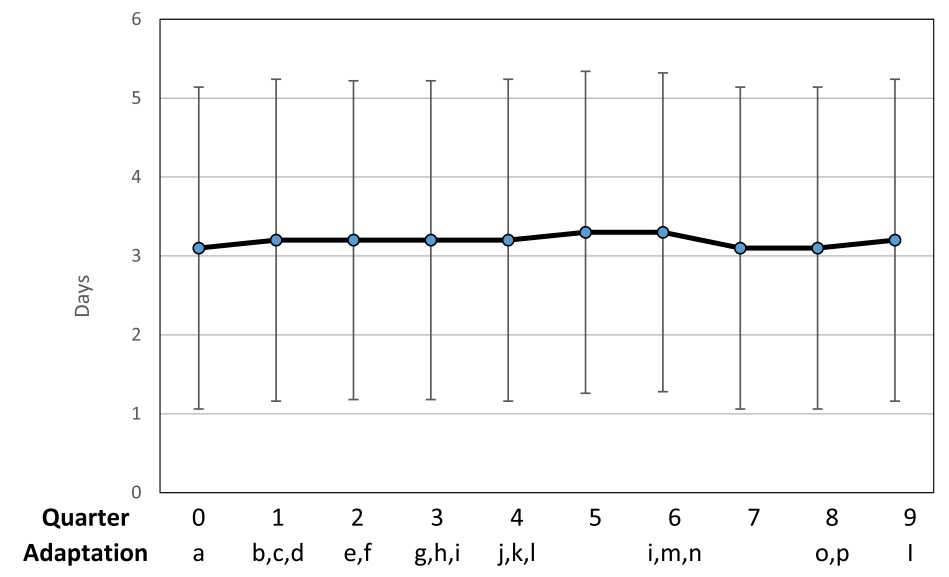

\begin{tabular}{|c|c|c|c|}
\hline \multicolumn{4}{|c|}{ Key } \\
\hline \multirow[b]{2}{*}{$\mathbf{a}$} & \multicolumn{3}{|l|}{ Adaptations } \\
\hline & $\begin{array}{l}\text { Addition of 30-day post-acute transition component to } \\
\text { the HaH model }\end{array}$ & $\mathbf{i}$ & $\begin{array}{l}\text { Expansion to new sites for enrollment along with } \\
\text { developing new intake procedures customized for } \\
\text { each site }\end{array}$ \\
\hline $\mathbf{b}$ & $\begin{array}{l}\text { Expansion of original target diagnoses and reduce } \\
\text { exclusions (e.g., HIV exclusion) to reflect current } \\
\text { medical practice }\end{array}$ & $\mathbf{j}$ & $\begin{array}{l}\text { Adaptation of intake procedure for patients } \\
\text { identified to need HaH services late at night by } \\
\text { holding the patients overnight in the emergency } \\
\text { department and transferring home in the morning }\end{array}$ \\
\hline c & Implementation of Palliative Care Unit at Home & $\mathbf{k}$ & $\begin{array}{l}\text { Launch of telehealth visits to supplement home } \\
\text { visits }\end{array}$ \\
\hline d & $\begin{array}{l}\text { Collaborated with community paramedicine program } \\
\text { to consult with } \mathrm{HaH} \text { physicians by video for patients } \\
\text { needing urgent visits in the home }\end{array}$ & l & $\begin{array}{l}\text { Internalized major portions of pharmacy and lab } \\
\text { services }\end{array}$ \\
\hline $\mathbf{e}$ & Contracting for infusion services & $\mathbf{m}$ & $\begin{array}{l}\text { Implemented new version of electronic medical } \\
\text { record }\end{array}$ \\
\hline $\mathbf{f}$ & Dedicated nurses hired & $\mathbf{n}$ & Dedicated physical therapist hired \\
\hline $\mathbf{g}$ & Implementation of Observation at Home & $\mathbf{0}$ & Role created for nurse care coordinator \\
\hline h & Implementation of Rehabilitation at Home & $\mathbf{p}$ & Piloted weekend admissions \\
\hline
\end{tabular}

Fig. 1 Mean Length of Stay (LOS) in days, Change over quarters SE 0.02, $p=0.75$ 


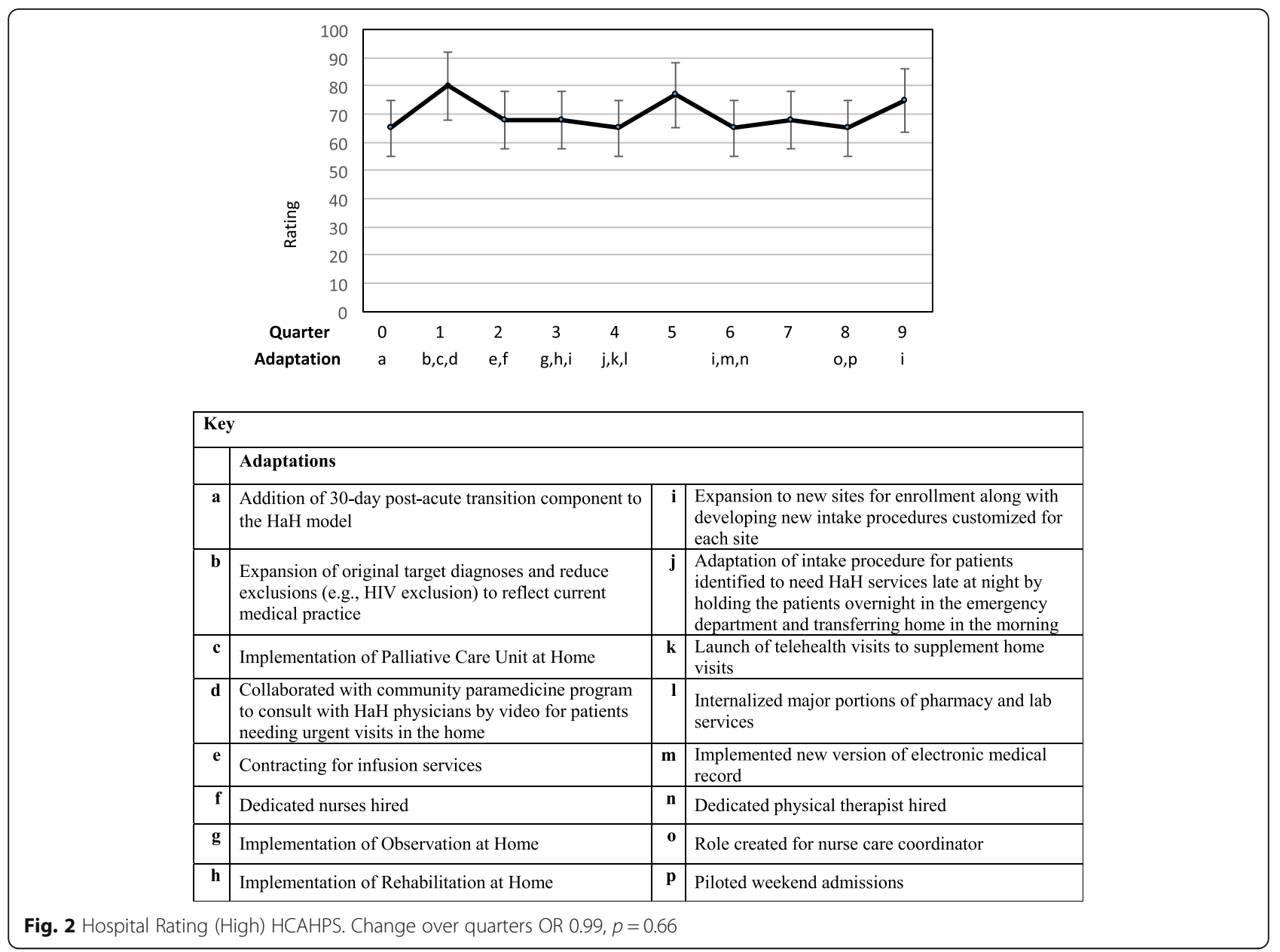

\section{Discussion}

We made adaptations to an evidence-based model of $\mathrm{HaH}$ at program inception and throughout a 33-month implementation. Our experience reinforces concepts from the DSF. Many adaptations were made to improve $\mathrm{HaH}$ operations. Others were made to respond to patient needs and referring provider requests such as for palliative care patients who needed acute hospital-level care. Some adaptations were made to improve scalability such as extending intake hours into nights and weekends. Other adaptations were made because new opportunities became available to enhance the patient experience such as the initiation of video telehealth and community paramedicine visits. These adaptations would have been difficult to include at program inception or even to script into phased introductions as the availability of the new opportunities or need for adaptation was unknown at that time. In some cases, adaptations were necessary to adapt to differing practice contexts at different hospitals. Expansion to other hospitals usually required new intake procedures to adjust to local culture, differing payer mix and social determinants of health in the surrounding community, and even different unions. The intervention also had to be adapted to payment and market forces affecting our hospitals (e.g., unanticipated creation and expansion of observation services during the period of implementation) and vendors (e.g., vendor budget issues, including vendor bankruptcy). We believe that the need and opportunity for dynamic adaptation occurs to varying degrees in the implementation of all new programs and is not limited to the $\mathrm{HaH}$ implementation experience we chart in this paper [2].

We modified the protocols used in our $\mathrm{HaH}$ program from the existing research evidence base described in the literature. For example, the addition of new diagnoses, alteration of clinical exclusions, and the addition of 30-day postacute follow up were changes from studied protocols. Despite this, for selected findings of LOS and patient satisfaction, our outcomes were similar to those reported in the original studies in Table 1 . Further, in a previous report [6], these outcomes over the period of implementation compared favorably to those of a comparison group of similar patients who were hospitalized in regular inpatient units. Thus, our implementation of 


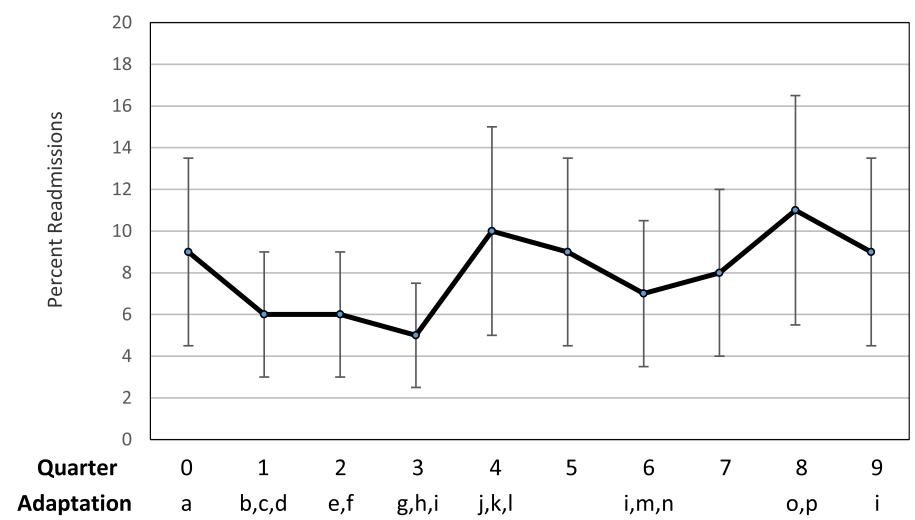

\begin{tabular}{|c|c|c|c|}
\hline \multicolumn{4}{|c|}{ Key } \\
\hline \multirow[b]{2}{*}{$\mathbf{a}$} & \multicolumn{3}{|l|}{ Adaptations } \\
\hline & $\begin{array}{l}\text { Addition of 30-day post-acute transition component to } \\
\text { the HaH model }\end{array}$ & i & $\begin{array}{l}\text { Expansion to new sites for enrollment along with } \\
\text { developing new intake procedures customized for } \\
\text { each site }\end{array}$ \\
\hline b & $\begin{array}{l}\text { Expansion of original target diagnoses and reduce } \\
\text { exclusions (e.g., HIV exclusion) to reflect current } \\
\text { medical practice }\end{array}$ & $\mathbf{j}$ & $\begin{array}{l}\text { Adaptation of intake procedure for patients } \\
\text { identified to need HaH services late at night by } \\
\text { holding the patients overnight in the emergency } \\
\text { department and transferring home in the morning }\end{array}$ \\
\hline c & Implementation of Palliative Care Unit at Home & $\mathbf{k}$ & $\begin{array}{l}\text { Launch of telehealth visits to supplement home } \\
\text { visits }\end{array}$ \\
\hline d & $\begin{array}{l}\text { Collaborated with community paramedicine program } \\
\text { to consult with } \mathrm{HaH} \text { physicians by video for patients } \\
\text { needing urgent visits in the home }\end{array}$ & $\mathbf{l}$ & $\begin{array}{l}\text { Internalized major portions of pharmacy and lab } \\
\text { services }\end{array}$ \\
\hline e & Contracting for infusion services & $\mathbf{m}$ & $\begin{array}{l}\text { Implemented new version of electronic medical } \\
\text { record }\end{array}$ \\
\hline f & Dedicated nurses hired & n & Dedicated physical therapist hired \\
\hline $\mathbf{g}$ & Implementation of Observation at Home & $\mathbf{0}$ & Role created for nurse care coordinator \\
\hline $\mathbf{h}$ & Implementation of Rehabilitation at Home & p & Piloted weekend admissions \\
\hline
\end{tabular}

Fig. 3 Percent Hospital Readmissions, Change over quarters, OR 1.00, $p=0.99$

an evidence-based intervention with its associated adaptations was accomplished with no obvious "program drift."

Additionally, we made adaptations in which we modified the $\mathrm{HaH}$ approach to changing context each quarter. For example, we added palliative care and other programs, as well as telehealth and community paramedicine visits. However, we did not observe significant changes in effectiveness across a variety of measures. LOS, 30-day readmissions, ED visits, and patient ratings of care did not significantly change over time. Tracking and reviewing these outcomes on a monthly basis as part of a continuous improvement activity may have been important in maintaining these effects through the implementation period. In the case of escalations, we observed a trend toward higher rates in later quarters. This was expected as our clinicians purposely started the program with less complex patients and enrolled increasingly complex patients as the team gained experience. The escalation rate was actually closely monitored throughout implementation, the program team was conscious of the trend, and cases of escalation were reviewed by the team on an ongoing basis in the interest of continuous improvement. Thus, we believe that the implementation of serial adaptations may have also avoided the phenomenon of "voltage drop."

Our study was limited by sample size by quarter. There are also occasional quarters where readmissions or other outcomes appear to increase. However, these occurrences are transient and the trend over the nine quarters is one of no change. A further limitation is that individual adaptations could be hypothesized to potentially enhance or diminish net benefits or program efficiency. For example, the community paramedicine intervention was intended to improve response time, enhance ability to adjust treatments in the home, and to reduce trips to the ED. One could hypothesize that this might enhance effectiveness. On the other hand, observation services at home were a departure from the evidence-based model, and one could hypothesize that these patients might be less likely to benefit. Because adaptations were made as the need or opportunity arose, it is not possible for us to isolate and examine the effect of any individual adaptation in this analysis. 


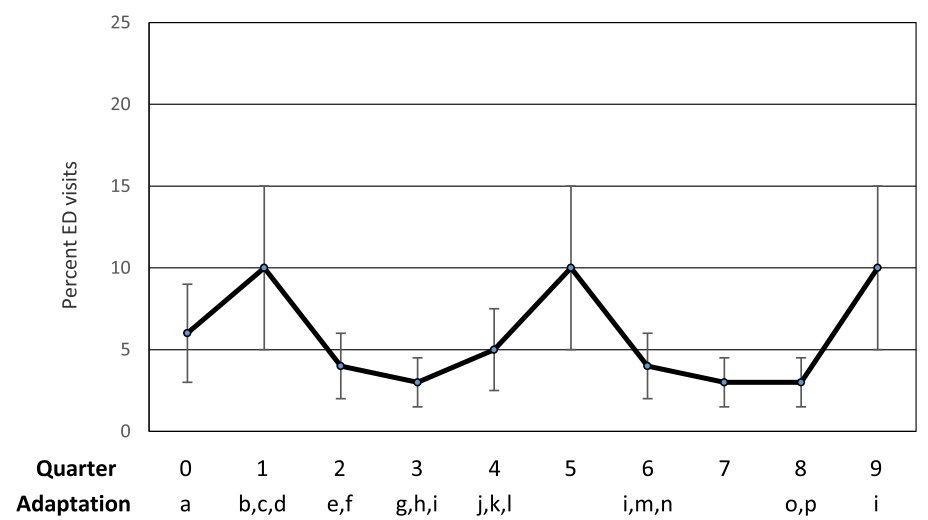

\begin{tabular}{|r|l|r|l|}
\hline \multicolumn{2}{|l|}{ Key } & Adaptations \\
\hline $\mathbf{a}$ & $\begin{array}{l}\text { Addition of 30-day post-acute transition component to } \\
\text { the HaH model }\end{array}$ & $\mathbf{i}$ & $\begin{array}{l}\text { Expansion to new sites for enrollment along with } \\
\text { developing new intake procedures customized for } \\
\text { each site }\end{array}$ \\
\hline $\mathbf{b}$ & $\begin{array}{l}\text { Expansion of original target diagnoses and reduce } \\
\text { exclusions (e.g., HIV exclusion) to reflect current } \\
\text { medical practice }\end{array}$ & j & $\begin{array}{l}\text { Adaptation of intake procedure for patients } \\
\text { identified to need HaH services late at night by } \\
\text { holding the patients overnight in the emergency } \\
\text { department and transferring home in the morning }\end{array}$ \\
\hline $\mathbf{c}$ & Implementation of Palliative Care Unit at Home & $\mathbf{k}$ & $\begin{array}{l}\text { Launch of telehealth visits to supplement home } \\
\text { visits }\end{array}$ \\
\hline $\mathbf{d}$ & $\begin{array}{l}\text { Collaborated with community paramedicine program } \\
\text { to consult with HaH physicians by video for patients } \\
\text { needing urgent visits in the home }\end{array}$ & $\mathbf{l}$ & $\begin{array}{l}\text { Internalized major portions of pharmacy and lab } \\
\text { services }\end{array}$ \\
\hline $\mathbf{e}$ & Contracting for infusion services & $\mathbf{m}$ & $\begin{array}{l}\text { Implemented new version of electronic medical } \\
\text { record }\end{array}$ \\
\hline $\mathbf{f}$ & Dedicated nurses hired & $\mathbf{n}$ & Dedicated physical therapist hired \\
\hline $\mathbf{g}$ & Implementation of Observation at Home & $\mathbf{0}$ & Role created for nurse care coordinator \\
\hline $\mathbf{h}$ & Implementation of Rehabilitation at Home & $\mathbf{p}$ & Piloted weekend admissions \\
\hline
\end{tabular}

Fig. 4 Percent ED Visits, Change over quarters, OR 0.93, $p=0.09$

\section{Conclusion}

The implementation of evidence-based programs necessarily requires adaptation. We made significant adaptations to $\mathrm{HaH}$ at inception and then serial adaptations over the course of implementation. Over the course of the implementation, many of these important outcomes were tracked and fed back to the program leadership leading to further adaptations. Our findings may indicate that adaptations to evidence-based programs may avoid diminished benefits due to potential 'program drift' or 'voltage drop'.

\section{Additional files}

Additional file 1: Hospital at Home Baseline Survey (containing self-rated health, demographic and functional status questions). (DOCX $20 \mathrm{~kb}$ )

Additional file 2: Hospital at Home Two-Week Survey (containing self-rated health, readmissions, ED visit and HCAHPS questions). (DOCX $29 \mathrm{~kb}$ )

Additional file 3: Hospital at Home Four-Week Survey (containing self-rated health, and functional status questions). (DOCX $18 \mathrm{~kb}$ )

Additional file 4: Regression models controlling for patient characteristics and season of admission mentioned in page 12. (DOCX $16 \mathrm{~kb}$ )

\section{Abbreviations}

ADL: Activities of Daily Living; Cl: Confidence Intervals; DSF: Dynamic Sustainability Framework; ED: Emergency Department; HaH: Hospital at Home; HCAHPS: Hospital Consumer Assessment of Healthcare Providers and Systems; ICMJE: International Committee of Medical Journal Editors; IRB: Institutional Review Board; LOS: Length of Stay; OR: Odds Ratio; PPHS: Program for the Protection of Human Subjects; SE: Standard Error

\section{Acknowledgements}

Not applicable.

\section{Funding}

Research reported in this publication was supported by the Department of Health and Human Services, Centers for Medicare \& Medicaid Services (1C1CMS331334-01-00), the National Institute on Aging Claude D. Pepper Older Americans Independence Center (TS, 3P30AG028741), and The John A. Hartford Foundation. The funders had no role in the design and conduct of the study; collection, management, analysis, and interpretation of the data; preparation, review, or approval of the manuscript; and decision to submit the manuscript for publication. The content of this paper is solely the responsibility of the authors and does not necessarily represent the official views of the U.S. Department of Health and Human Services or any of its agencies. The research presented was conducted by the awardee. Findings may or may not be consistent with or confirmed by the findings of the independent evaluation contractor. 


\section{Availability of data and materials}

The datasets generated and/or analyzed during the current study are not publicly available due to the inclusion of protected health information but are available from the corresponding author on reasonable request.

\section{Authors' contributions}

ALS, MG, LVD, BL and BM conceived and designed the study. ALS and RMZ drafted the manuscript. ADF, LVD, MG, BM, and BL provided critical revision of the manuscript for important intellectual content. ADF, SL, and EC provided statistical analysis. ALS, LVD, and BL obtained funding. BM, SL, and EC provided administrative, technical and material support. ALS and ADF provided supervision. All authors read and approved the final manuscript, each author has participated sufficiently in the work to take public responsibility for appropriate portions of the content and agreed to be accountable for all aspects of the work

\section{Ethics approval and consent to participate}

Ethics approval was granted by the Icahn School of Medicine at Mount Sinai's Program for the Protection of Human Subjects (ID number IRB-17-02565) for this retrospective analysis of data. The approval included a waiver of informed consent for data that were collected for grant reporting, quality assurance, and internal program monitoring purposes.

\section{Consent for publication}

Not applicable.

\section{Competing interests}

$A L S, A F, L V D, B M, S L$, and EC are full time employees of the Icahn School of Medicine, which in turn has an ownership interest in a joint venture with Contessa Health, a venture that manages acute care services provided to patients in their homes through prospective bundled payment arrangements. $A L S, A F, L V D, B M, S L$, and $E C$ have no personal financial interest in the joint venture. Authors RZ, MG, and BL have no competing interests.

\section{Publisher's Note}

Springer Nature remains neutral with regard to jurisdictional claims in published maps and institutional affiliations.

\section{Author details}

'Brookdale Department of Geriatrics and Palliative Medicine, Icahn School of Medicine at Mount Sinai, 1 Gustave L. Levy Place, New York, NY 10029, USA. ${ }^{2}$ Icahn School of Medicine at Mount Sinai, New York, USA. ${ }^{3}$ Division of General Internal Medicine, Department of Medicine, Icahn School of Medicine at Mount Sinai, New York, NY, USA. ${ }^{4}$ Division of Geriatrics, Department of Medicine, Johns Hopkins University School of Medicine, Baltimore, Maryland, USA. ${ }^{5}$ James J. Peters VA Medical Center, New York, NY, USA. 'Department of Health Law, Policy \& Management, Boston University School of Public Health, Boston, Massachusetts, USA.

Received: 20 August 2018 Accepted: 5 April 2019

Published online: 29 April 2019

\section{References}

1. Stirman SW, Miller CJ, Toder K, Calloway A. Development of a framework and coding system for modifications and adaptations of evidence-based interventions. Implement Sci. 2013;8:65.

2. Aarons GA, Green AE, Palinkas LA, Self-Brown S, Whitaker DJ, Lutzker JR, et al. Dynamic adaptation process to implement an evidence-based child maltreatment intervention. Implement Sci. 2012;7:32.

3. Chambers DA, Glasgow RE, Stange KC. The dynamic sustainability framework: addressing the paradox of sustainment amid ongoing change. Implement Sci. 2013;8:117.

4. Chambers DA, Norton WE. The Adaptome: advancing the science of implementation adaptation. Am J Prev Med. 2016;51:4.

5. Leff B, Burton L, Mader SL, Naughton B, Burl J, Inouye SK, et al. Hospital at home: feasibility and outcomes of a program to provide hospital-level care at home for acutely III older patients. Ann Intern Med. 2005;143:11.

6. Federman AD, Soones T, Decherrie LV, Leff B, Siu AL. Association of a bundled hospitalat-home and 30-Day postacute transitional care program with clinical outcomes and patient experiences. JAMA Intern Med. 2018;178:1033.
7. Stessman J, Ginsberg G, Hammerman-Rozenberg R, Friedman R, Ronen D, Israeli A, et al. Decreased hospital utilization by older adults attributable to a home hospitalization program. J Am Geriatr Soc. 1996;44:5.

8. Wilson A, Parker H, Wynn A, Jagger C, Spiers N, Jones J, et al. Randomised controlled trial of effectiveness of Leicester hospital at home scheme compared with hospital care. BMJ. 1999;319:1542.

9. Caplan GA, Ward JA, Brennan NJ, Coconis J, Board N, Brown A. Hospital in the Home: a randomised control trial. Med J Aust. 1999;170:4.

10. Harris R, Ashton T, Broad J, Connolly G, Richmond D. The effectiveness, acceptability and costs of a hospital-at-home service compared with acute hospital care: a randomized controlled trial. J Health Serv Res Policy. 2005;10:3.

11. Cryer L, Shannon SB, Van Amsterdam M, Leff B. Costs for "hospital at home" patients were 19 percent lower, with equal or better outcomes compared to similar inpatients. Health Aff. 2012;31:6.

12. Summerfelt WT, Sulo S, Robinson A, Chess D, Catanzano K. Scalable hospital at home with virtual physician visits: pilot study. Am J Manag Care. 2015;21:10.

13. Center for Medicare and Medicaid Services. HCAHPS: Patients' Perspectives of Care Survey. 2011. https://www.cms.gov/Medicare/Quality-Initiativespatient-assessment-instruments/hospitalqualityinits/hospitalhcahps.html. Accessed 20 Feb 2018.

\section{Ready to submit your research? Choose BMC and benefit from:}

- fast, convenient online submission

- thorough peer review by experienced researchers in your field

- rapid publication on acceptance

- support for research data, including large and complex data types

- gold Open Access which fosters wider collaboration and increased citations

- maximum visibility for your research: over $100 \mathrm{M}$ website views per year

At $\mathrm{BMC}$, research is always in progress.

Learn more biomedcentral.com/submissions 